\title{
Blockchain Implications in the Management of Patient Complaints in Healthcare
}

\author{
Yazan M. Alkhateeb \\ Clinical Governance Department, American Hospital Dubai, Dubai, UAE \\ Email: yazalkhateeb@gmail.com,yalkhateeb@ahdubai.com
}

How to cite this paper: Alkhateeb, Y.M. (2021) Blockchain Implications in the Management of Patient Complaints in Healthcare. Journal of Information Security, 12, 212-223.

https://doi.org/10.4236/jis.2021.123011

Received: April 24, 2021

Accepted: June 4, 2021

Published: June 7, 2021

Copyright ( 2021 by author(s) and Scientific Research Publishing Inc. This work is licensed under the Creative Commons Attribution International License (CC BY 4.0).

http://creativecommons.org/licenses/by/4.0/

(c) (i) Open Access

\begin{abstract}
Blockchain is an emerging technology that has recently been the focus for many researchers who have highlighted its diverse applications including healthcare. Transparency in managing unsolicited patient complaints is important in healthcare for both patients and healthcare providers; in addition, patient complaints analysis is significant to the continued quality improvement. Accordingly, the purpose of this study is to understand the nature of patient complaints management in the healthcare settings, explore the implications of blockchain on the management of patient complaints, and identify limitations in the usage of blockchain. Structured qualitative review and content analysis of the literature methods were used through multiple inclusion and exclusion phases for the scope of this research. Blockchain technology characteristics have been analyzed and approximated with desired features in the patients' complaint management. Patient complaints provide valuable information to drive continuous improvements in healthcare. Blockchain is described as transparent, decentralized, immutable and anonymous. Results of this research found that a complaint Management system that is built on blockchain technology might have desired features that involve data integrity, security and transparency. Blockchain does have certain limitations that involve cybersecurity, scalability, confidentiality, readiness to adopt it, and is uncertainty about its impact. As a conclusion, implementing a system to manage patient complaints that is based on blockchain technology is promising, due to its desired possible features.
\end{abstract}

\section{Keywords}

Blockchain, Unsolicited Patient Complaints, Health Care, Transparency

\section{Introduction}

Nearly half of the global population does not have access to the basic and essen- 
tial healthcare they need [1]. In the European Union 9.6\% of the GDP is spent on healthcare, where the highest was in Germany and France reaching 11\%, and lower than 6\% in Romania in 2017 [2].

In 2016, there were around 145 million patient visits to the emergency department, in the USA [3]. Patients expect their health-related issues to be dealt with in a caring and timely manner and to receive a high quality of care. However, $40 \%$ of patients, who visit primary and ambulatory healthcare settings are affected by inadequate safety conditions, leading them to be admitted for further care, which could have been avoided [4]. In response to the poor quality of care and services they have received, patients or their families will be unsatisfied, resulting in a complaint to the health care facility or to the regulatory bodies [5].

It is important to involve the voice of patients as a contributor to the process of continuous Quality Improvement (CQI). Managers in healthcare should not rely on intra-organizational initiatives only for CQI, but they should incorporate the patient's perspective, as presented in patient complaints and surveys [6]. Thereby the valuable insights that can be deemed from analysis of patient complaints can be employed for CQI in healthcare.

PwC's Global Blockchain Survey evidences that $11 \%$ of healthcare organizations are already gaining significant benefit from deployment of blockchain technology [7]. The report revealed that for healthcare firms, blockchain technology can provide several opportunities. For example, it can transform the data collection procedure, as well as data distribution, data monitoring and leveraging of data for future use [7]. Therefore, blockchain adoption in healthcare is expected to increase in the future.

The existing literature has provided insights about complaints management in healthcare and highlighted the importance of applying system-wide approach in patient complaint management. Research studies that focused on healthcare complaint management highlighted certain steps, strategies or best practices that can be applied in patient complaint handling. It was noted in the literature that patient complaints were widely used as a continuous quality improvement tool to identify gaps in the healthcare delivery system [8] [9] [10], other studies focused on creating coding for the patient complaints, which showed similarity on the topics patients are complaining about, In addition, it was concluded in the literature that there's a lack of implementing patient complaints management as a system-wide approach [11] [12]. Although the existing literature discussed the usage of blockchain in healthcare, it did not explore the implications of using such technology in the patient complaints management.

The aim of this research is to evidence this author's hypothesis that blockchain will enable improvements in the management of patient complaints, highlighting the limitations and challenges that have been identified and ways that this hypothesis can be tested in application of blockchain to patient complaints management.

The research seeks to address the following research questions.

1) What is the nature of complaint management in the healthcare setting? 
2) What are the implications of adopting blockchain technology in the patient complaints management?

3) What are the limitations of using blockchain technology?

\section{Methods}

Industry standard quality improvement models were reviewed including: Root cause analysis RCA [13]; Failure Modes and Effects Analysis FMEA [14]; Plan Do Check Act PDCA [15]; and Continues Quality Improvement CQI [16].

Further to this, a structured qualitative review and content analysis of the literature that is related to blockchain and complaints management in healthcare was conducted, by performing the following steps:

1) Search keywords were identified that include, blockchain, block chain, patient complaints, complaints, complaint management in healthcare, unsolicited patient complaints.

2) Keywords were used separately and in combination in different databases including: ScienceDirect; Emerald Insight; IEEE; Springer Link; EBSCO; Google Scholar.

3) The search was initially defined to include articles that discuss unsolicited patient complaints. The word complaint is widely used in healthcare, to describe the presenting clinical problem of a patient, known as chief complaints. To further refine, titles were reviewed to exclude articles that related to the presenting clinical complaints of a patients.

4) Inlusion and exclusion criteria in selecting research topics (Inclusion: Unsolicited Patient complaints, patient complaints in healthcare, Unsolicited patient complaints in healthcare, Written in English), (exclusion: Patient chief complaints, Patient presenting complaints, Presenting problems, presenting complaint)

5) A further stage of research, the same search criteria were applied to the abstracts of the collected articles. The articles were studied to explore the implications of adopting blockchain characteristics for patient complaints management.

6) The collected literature content was studied and critically analzyed on how to employ the characterstics of blockchain technology in the patient complaints managment and explore their impact on the patient complaints desired features.

\section{Literature Review}

\subsection{Complaints in Healthcare}

Patient complaints management has received attention in order to monitor quality and patient safety [11]. Understanding patient complaints provides useful insight into the effect of safety-related issues in healthcare provision. In addition, analysing the problems patients face helps the organization to understand the systematic challenges in the healthcare setting [11]. For example, the Francis report [17] in the UK, that was published based on a public inquiry into poor care at the Mid Staffordshire NHS Foundation Trust, highlighted that there have 
been 1200 deaths as a result of unacceptable neglect, Additionally as found by the report, the hospital neglected the patient complaints throughout the duration of such incidents, which resulted in a poor level of trust among the patients and impacted the image of the hospital

Healthcare organizations, on a day-to-day basis receive huge volumes of grievances and complaints that relate to diverse issues and problems [11]. In England, the number of complaints submitted to the NHS between 1st of April 2018 and March 31st 2019 was 208,924 written complaints, representing 4018 complaints per week [18]. These figures suggest that patient complaints management is a very serious matter that warrants careful handling. Moreover, since the complaints are varied and diverse in nature it is important to have a stringent system that can manage complaints in healthcare services. However, complaints in different regions are not systematically recorded or categorized for future reference which prevents analysis or understanding of the key drivers of complaint [11] [19].

Attempting to define quality of care from the patient perspective, prior research has proposed that it is the ability to get the treatment without difficulty and actually benefit from it that denotes a high quality of care provision [20]. A survey conducted with the aim to explore the reason why patients complain, concluded that patients raise complaints to achieve justice and explanation from both the treating health care provider and the hospital additionally to prevent of harm from happening to others [21]. Similarly in a study by Jangland, Gunningberg [22] found that the key expectation of patients in making their complaint was that the hospital would have a fair and transparent complaints management system. This evidences the need for an open, transparent system for complaint and procedures to respond accordingly.

Complaints are an important measure of the quality level provided because they reflect the patient's experience and can be used as quality improvement tool. Patient's complaint texts can be of a valuable source of insight, for quality enhancement and educational programs, which will minimize communication gaps between patients and healthcare providers [22].

This author proposes that a standardized classification schema complaint should be appended to healthcare ontology such as SNOMED widely in use globally to record clinical events and define optimal care pathways. Importantly this will allow complaints data to be stored alongside patient records and accessed alongside ongoing patient management. In addition, this will enable systematic analysis of aggregate patient complaints data to understand who complains about what [11]. It is unquestionable that systematically recording and managing patient complaints are important in improving healthcare provision, but this systematic approach will also enable to explore whether complaints as a patient "event", subsequently have any impact on clinical outcomes.

\subsection{Blockchain}

Blockchain gained attention when the bitcoin was created [23]. Blockchain tech- 
nology was first described in a white paper by Satoshi Nakamoto and the technology was potentially groundbreaking for bitcoin cryptocurrency [24]. Blockchain solved the risk of double spending, whereby digital currency might effectively be spent more than once as a result of fraudulent replication [25]. Physical currencies cannot be easily replicated, and the parties involved in a transaction can easily verify authenticity and past ownership.

Blockchain technology has gained attention to be used in different applications such as smart contracts [24]. A blockchain is comprised of digital records that interact and are exchanged, storing the transaction data in chronological order [26]. The name was driven from its natural architecture, wherein blocks are recorded in sequence and connected on a cluster rundown, creating a chain [27] [28] [29]. Satoshi created trust in distributed ledger systems, where any tampering of the data registered would be detected.

Similarly, Beck [30] describes blockchain as a tamper-proof database that runs through nodes while performing transactions, the cryptography capabilities verify and authenticate data transactions through a hash function. The hash function works as an authentication for the previous recorded data for each unique transaction [24]. Hence blockchain provides data exchange in a secure manner that verifies and records data chronologically.

Each transaction that is added to the chain is verified by different devices on the same network and administered by a structured framework. This framework will control and verify exchanges of data, authenticating any additions, making sure that no unacceptable inputs or squares will be added to the chain, and that the chain keeps its integrity as one structured form [28]. Blockchain technology can be used when taking into consideration the integrity of data and information [31].

Blockchain is used in cryptocurrency for transactions such as the bitcoin, however it has different applications in other areas as well [23] [27] [32]. Blockchain technology can be applied to any digital data format that is exchanged and processed online [28].

\subsection{Blockchain in Healthcare}

Healthcare organizations have witnessed major challenges due to traditional manual processes that are slow-moving, further complicating data-driven processes and procedures. Such systems can be made more efficient by implementing blockchain technology, leading to more effective business operations. As noted by $\mathrm{PwC}$ Health Research Institute [33] Blockchain adoption can also be seen in pharma inventory management systems, whereby it can help identify transaction history.

Blockchain technology also enables patients to share their personal medical information with physicians [34]. With greater privacy and security [35], the robust security system in blockchain technology helps firms monitoring compliance information and reporting any deviations from the set guidelines. There is clear evidence then that blockchain has significant applicability in healthcare data management and can be implemented in managing patient complaint data. 
Blockchain technology thus, has a promising future in health care applications which will boost the efficiency and transparency of the status quo; however it's still only emergent in maturity, and requires careful incremental steps to understand the accompanied safety and scalability risks from applying this technology [36]. Mettler [37] has concluded that blockchain has an imminent future in healthcare and will create disruption in service provision by accelerating digital transformation.

According to Kuo, et al. [25] the main benefit from using blockchain in healthcare and biomedical applications is the decentralized management of data, allowing healthcare stakeholders such as hospitals and government regulatory bodies to cooperate and share data without having one central intermediary. Additionally, immutably, robustness, and security are considered the main benefits of blockchain adopted in healthcare leading to improved data integrity of data, and more efficient

\section{Characteristics of Blockchain}

\subsection{Transparency}

The bases of blockchain are in its characteristics of trustworthiness and transparency. The blocks have to verify and agree on the information for the retrospective data, any modification or changes will be detected by the concerned parties involved, so that any corruption of data will be detected [30]. Blockchain can also be used to reduce fraud and provide transparency in transactions other than financial applications, such as the ownership of lands and property's proposed transfer project in Georgia and Honduras [23].

The usage of this feature in the context of healthcare is significant. In their study Benchoufi and Ravaud [38] concluded that the transparency characteristic of blockchain can be very helpful in clinical trial research, where it will help make the process of clinical trials more structured, transparent, and reliable.

In addition, Mettler [37] also noted that healthcare organizations have a number of stakeholders (suppliers, patients, healthcare professionals, doctors, government) that create a unified healthcare ecosystem. Within this ecosystem blockchain technology can provide all the medical stakeholders access to treatment information and other regulatory and compliance matters. This can enhance transparency and enhance the process of quality management that is deemed essential in healthcare service delivery.

Both, the patients and healthcare providers desire that the complaints to be dealt in fair and transparent way, it's concluded in the view of this author that adopting blockchain in complaints management will have a role in generating trust, by increasing the transparency of data managed throughout the patient complaint management process.

\subsection{Anonymity}

According to Zheng, et al. [39] anonymity is one of the significant features of 
blockchain technology, additionally as noted by the authors; interactions between the users of blockchain technology can happen through an automatically generated address that prevents identification of the user. Furthermore, Zheng, et al. [39] added that users can generate multiple addresses to mask their identity, since there is no central party who keeps the private information of the users there is a certain degree of privacy of transactions conducted on the blockchain platform. This feature can thus have its significant applicability in healthcare complaint management.

In conclusion, patient complaints can drive continues quality improvement, and in order to encourage patients to register their complaints, even if they have consideration about their identity revealed in reporting issues in the healthcare system, it is the view of this author that using a blockchain-based complaint management system, the patients can potentially register complaints that will be anonymous and enhance privacy of the individuals' medical issues.

\subsection{Decentralized}

Blockchain data are not stored on one single device. Where it is not based on a single node, it is rather shared on different devices and systems [26]. Well-known cloud-based storage services provided such as Google Drive, Dropbox are used widely despite the risk of privacy breaches or data control. However blockchain peer to peer distributed storage, provides a decentralized platform to store data based on blockchain without relying on a third party this latter environment will more likely increase security, data integrity and privacy [28].

In a centralized platform each transaction has to go through a central entity for verification, but blockchain technology provides a decentralized approach where it removes the arbitrator and connects directly to other parties [40]. According to Mettler [37] this has significant advantages in the context of healthcare organizations. For instance, in the medical treatment process such technologies can create added value by providing multiple stakeholders with access to the same information. Mettler [37] also noted that it can prevent errors, by reducing medical negligence that occurs due to outdated information.

In summary, such technologies can help create a system-wide approach whereby complaints registered can be accessed by multiple players involved and thus, provide for its speedy remedy. The author concludes that these features of blockchain will have significant applicability in patient complaint management.

Continues quality improvement results from the learnings gathered by analyzing patient complaints, not just within one facility only, the lessons learned from one entity can be shared so other healthcare facilities can learn from it, with the support from regulator to make sure these learned lessons are shared in a context that guarantee the privacy and anonymity of these data. This author proposes that the decentralized nature of blockchain technology could facilitate greater collaboration among the healthcare entities and regulatory bodies. This approach has not been highlighted by prior research studies and warrants further exploration. 


\subsection{Immutability}

A noteworthy and unique feature of blockchain technology is its ability to be unaffected by any modification and alteration [41]. Similarly Niranjanamurthy, et al. [26] stated that blockchain transactions are immutable, meaning that they cannot be changed or modified. However, blockchain data is not fully tamper proof as mentioned in different papers. The stored data in blockchain is tamper-proof and stored forever unless more than $51 \%$ of the nodes are acquired [26].

Similarly Yaga, et al. [42] mentioned that permission-less blockchain networks are in risk of an attack that will allow other entities to take control of the data. However, in a permissioned blockchain data network this problem of attack can be mitigated. In their study Gordon and Catalini [43] mentioned that by implementing a permissioned blockchain the healthcare organizations can establish control membership to access, read, or write. It also enhances interoperability in the patient-care context.

Gordon and Catalini [43] highlight that previously patients have been solely reliant on institution-driven data. However, with such an advanced system in place, patients could be in-charge of generating and managing information. As can be understood from this discussion, such permissioned blockchain can help enhancing patient-centric care and provide them opportunity to manage their own data.

Patients and health care providers expect that the complaint management systems are free from any data tampering related to the complaint, or the data collected and interpreted during the complaint management process itself. This author proposes the hypothesis that if we apply blockchain in the context of complaint handling, immutability will enhance the accuracy of the complaint data and patients will be able to access such data with greater confidence. In addition the data will be tamper proof, and any modification to data will be detected.

\section{Analysis}

\subsection{Desired Features}

Although the existing literature highlighted the significant applicability of blockchain in healthcare it has not yet clearly analyzes how the features of blockchain like immutability, transparency, anonymity, and decentralization can be implemented in healthcare service delivery to tackle patient complaints and quality improvements.

This author proposes that a patient complaints management system that comprises blockchain features might help patients register complaints, safeguard their privacy and ensure data accuracy throughout the process of managing patient complaints.

This study provides a step in exploring the possibility of implementing blockchain in the management of patient complaints. Building a system to manage patient complaints that is based on blockchain should consider the limitation of 
blockchain, being an emergent technology.

\subsection{Blockchain in Patient Complaints Management}

Key advantages of blockchain technology are process integrity, immutability and transparencies, which will be very helpful, since that resolving a complaint in healthcare go through a structured process until reaching the final outcome or conclusion, which will be provided to the patient and the healthcare providers. In the course of this process, data integrity, security and immutability does safeguard the rights of patients and the healthcare providers since no data related to the complaint such as complaint letter, health records, and diagnostic reports will be tampered, and if happened it will be detected. blockchain will likely make the complaints management more secured, transparent and have impact on the integrity of the patient complaint process.

\subsection{Blockchain Limitations}

Despite the applicability of blockchain technology, it does have certain limitations. Blockchain is a new technology, and it requires incremental progress in adoption. Thorough assessment of challenges such as scalability, confidentiality and the threat of attack are advised before adopting this technology in any field [25]. Niranjanamurthy, et al. [26] concluded that there are multiple risks in adopting blockchain technology such as privacy and security issues, government caution due to the regulatory impact and uncertainty about the impact. Furthermore Yaga, et al., [42] mentioned that malicious users and cyberattacks can take over the control of blockchain networks, resulting in privacy breaches and loss of data.

A challenge that might be faced if the management of patient complaints is built on a private blockchain rather than open blockchain, where it will be only partially decentralized. Tampering of data is possible, however tampering would be detected retrospectively as a proof of existence [40].

In summary, although blockchain technology has certain limitations in terms of security issues, it can significantly drive the process of data management in the healthcare system. The features of immutability, transparency can ensure that complaints from the patients are registered accurately and are addressed in a timely and efficient manner. In terms of quality enhancement, it can also make a significant contribution to ensure greater collaboration among healthcare professionals.

\section{Conclusion \& Future Work}

Managing patient complaints in healthcare is very important and will help improve the services provided. Implementing blockchain technology in patient complaints management is promising, due to the characteristics of blockchain that likely will positively impact the features of healthcare complaints management. 
Future work will be on research in this topic and creation of a live test environment will provide more understanding and insights. Implementing the system should be incremental to assess the impact on patient complaints management while considering the limitations and risks of blockchain.

\section{Conflicts of Interest}

The author declares no conflicts of interest regarding the publication of this paper.

\section{References}

[1] WHO (2017) Tracking Universal Health Coverage: 2017 Global Monitoring Report. World Health Organization and International Bank for Reconstruction. https://apps.who.int/iris/bitstream/handle/10665/259817/9789241513555-eng.pdf;js essionid=4FFADCC707FC6349E628905E58A5F2A5 sequence $=1$

[2] OECD/EU (2018) Health at a Glance: Europe 2018: State of Health in the EU Cycle. OECD Publishing, Paris.

https://www.oecd-ilibrary.org/social-issues-migration-health/health-at-a-glance-eur ope-2018_health_glance_eur-2018-en

[3] CDC (2017) Centers for Disease Control and Prevention. https://www.cdc.gov/nchs/data/hus/hus17.pdf

[4] Auraaen, A., Slawomirski, L. and Klazinga, N. (2018) Economics of Patient Safety in Primary and Ambulatory Care. OECD, Paris.

[5] Bendall-Lyon, D. and Powers, T.L. (2001) The Role of Complaint Management in the Service Recovery Process. The Joint Commission Journal on Quality Improvement, 27, 278-286. https://doi.org/10.1016/S1070-3241(01)27024-2

[6] Javetz, R. and Stern, Z. (1996) Patients' Complaints as a Management Tool for Continuous Quality Improvement. Journal of Management in Medicine, 10, 39-48. https://doi.org/10.1108/02689239610122306

[7] PwC (2018) Global Blockchain Survey, 2018. https://www2.deloitte.com/tr/en/pages/financial-services/articles/2018-global-block chain-survey.html

[8] Cooper, W.O., Martinez, W., Domenico, H.J., Callahan, S.T., Kirkby, B.P., Finlayson, A.J., et al. (2018) Unsolicited Patient Complaints Identify Physicians. The American Journal of Geriatric Psychiatry, 26, 927-936.

https://doi.org/10.1016/j.jagp.2018.04.005

[9] Kee, J.W., Khoo, H.S., Lim, I. and Koh, M.Y. (2018) Communication Skills in Patient-Doctor Interactions: Learning from Patient Complaints. Health Professions Education, 4, 97-106. https://doi.org/10.1016/j.hpe.2017.03.006

[10] Gallagher, T.H. and Mazor, K.M. (2015) Taking Complaints Seriously: Using the Patient Safety Lens. BMJ Quality \& Safety, 24, 352-355.

https://doi.org/10.1136/bmjqs-2015-004337

[11] Reader, T.W., Gillespie, A. and Roberts, R. (2014) Patient Complaints in Healthcare Systems: A Systematic Review and Coding Taxonomy. BMJ Quality \& Safety, 23, 678-689. https://doi.org/10.1136/bmjqs-2013-002437

[12] Harrison, R., Walton, M., Healy, J., Smith-Merry, J. and Hobbs, C. (2016) Patient Complaints about Hospital Services: Applying a Complaint Taxonomy to Analyse and Respond to Complaints. International Journal for Quality in Health Care, 28, 
240-245. https://doi.org/10.1093/intqhc/mzw003

[13] Rooney, J.J. and Heuvel, L.N.V. (2004) Root Cause Analysis for Beginners. Quality Progress, 37, 45-56.

[14] Stamatis, D.H. (2003) Failure Mode and Effect Analysis: FMEA from Theory to Execution. ASQ Quality Press.

[15] Moen, R. and Norman, C. (2006) Evolution of the PDCA Cycle.

[16] Shortell, S.M., O’Brien, J.L., Carman, J.M., Foster, R.W., Hughes, E.F., Boerstler, H., et al. (1995) Assessing the Impact of Continuous Quality Improvement/Total Quality Management: Concept versus Implementation. Health Services Research, 30, 377.

[17] Report F. (2013) Report of the Mid Staffordshire NHS Foundation Trust Public Inquiry: Executive Summary (Vol. 947). The Stationary Office, London.

[18] NHS (2019) National Health Service. https://digital.nhs.uk/data-and-information/publications/statistical/data-on-written -complaints-in-the-nhs

[19] O’Donovan, O. and Madden, D. (2018) Why Do Medical Professional Regulators Dismiss Most Complaints from Members of the Public? Regulatory Illiteracy, Epistemic Injustice, and Symbolic Power. Journal of Bioethical Inquiry, 15, 469-478. https://doi.org/10.1007/s11673-018-9869-2

[20] Campbell, S.M., Roland, M.O. and Buetow, S.A. (2000) Defining Quality of Care. Social Science \& Medicine, 51, 1611-1625. https://doi.org/10.1016/S0277-9536(00)00057-5

[21] Friele, R.D. and Sluijs, E.M. (2006) Complaint Handling in Hospitals: Empirical Data. BMC Health Services Research, 6, 106.

https://doi.org/10.1186/1472-6963-6-106

[22] Jangland, E., Gunningberg, L. and Carlsson, M. (2009) Patients' and Relatives' Complaints about Encounters and Communication in Health Care: Evidence for Quality Improvement. Patient Education and Counseling, 75, 199-204. https://doi.org/10.1016/j.pec.2008.10.007

[23] Underwood, S. (2016) Blockchain beyond Bitcoin. Communications of the ACM, 59, 15-17. https://doi.org/10.1145/2994581

[24] Di Pierro, M. (2017) What Is the Blockchain? Computing in Science \& Engineering, 19, 92-95. https://doi.org/10.1109/MCSE.2017.3421554

[25] Kuo, T.T., Kim, H.E. and Ohno-Machado, L. (2017) Blockchain Distributed Ledger Technologies for Biomedical and Health Care Applications. Journal of the American Medical Informatics Association, 24, 1211-1220. https://doi.org/10.1093/jamia/ocx068

[26] Niranjanamurthy, M., Nithya, B.N. and Jagannatha, S. (2018) Analysis of Blockchain Technology: Pros, Cons and SWOT. Cluster Computing, No. 5, 1-15. https://doi.org/10.1007/s10586-018-2387-5

[27] Meunier, S. (2018) Blockchain 101: What Is Blockchain and How Does This Revolutionary Technology Work? In: Transforming Climate Finance and Green Investment with Blockchains, Academic Press, Cambridge, 23-34. https://doi.org/10.1016/B978-0-12-814447-3.00003-3

[28] Crosby, M., Pattanayak, P., Verma, S. and Kalyanaraman, V. (2016) Blockchain Technology: Beyond Bitcoin. Applied Innovation, 2, 71.

[29] Iansiti, M. and Lakhani, K.R. (2017) The Truth about Blockchain. Harvard Business Review, 95, 118-127. 
[30] Beck, R. (2018) Beyond Bitcoin: The Rise of Blockchain World. Computer, 51, 54-58. https://doi.org/10.1109/MC.2018.1451660

[31] Lemieux, V.L. (2016) Trusting Records: Is Blockchain Technology the Answer? Records Management Journal, 26, 110-139.

https://doi.org/10.1108/RMJ-12-2015-0042

[32] Conoscenti, M., Vetro, A. and Martin, D. (2016) Blockchain for the Internet of Things: A Systematic Literature Review. IEEE/ACS 13 th International Conference of Computer Systems and Applications (AICCSA), Agadir, 29 November-2 December 2016, 1-6. https://doi.org/10.1109/AICCSA.2016.7945805

[33] PWC (2018) A Prescription for Blockchain and Healthcare: Reinvent or Be Reinvented.

https://www.pwc.com/mx/es/publicaciones/archivo/2018/10/20181010-pwc-mx-a-p rescription-for-blockchain-and-healthcare.pdf

[34] Yue, X., Wang, H., Jin, D., Li, M. and Jiang, W. (2016) Healthcare Data Gateways: Found Healthcare Intelligence on Blockchain with Novel Privacy Risk Control. Journal of Medical Systems, 40, 218. https://doi.org/10.1007/s10916-016-0574-6

[35] Xia, Q., Sifah, E., Smahi, A., Amofa, S. and Zhang, X. (2017) BBDS: Blockchain-Based Data Sharing for Electronic Medical Records in Cloud Environments. Information, 8, 44-56. https://doi.org/10.3390/info8020044

[36] Angraal, S., Krumholz, H.M. and Schulz, W.L. (2017) Blockchain Technology: Applications in Health Care. Cardiovascular Quality and Outcomes, 10, e003800. https://doi.org/10.1161/CIRCOUTCOMES.117.003800

[37] Mettler, M. (2016) Blockchain Technology in Healthcare: The Revolution Starts Here. IEEE 18th International Conference on e-Health Networking, Applications and Services (Healthcom), Piscataway, 14-16 September 2016, 1-3. https://doi.org/10.1109/HealthCom.2016.7749510

[38] Benchoufi, M. and Ravaud, P. (2017) Blockchain Technology for Improving Clinical Research Quality. Trials, 18, 335. https://doi.org/10.1186/s13063-017-2035-Z

[39] Zheng, Z., Xie, S., Dai, H.N., Chen, X. and Wang, H. (2018) Blockchain Challenges and Opportunities: A Survey. International Journal of Web and Grid Services, 14, 352-375. https://doi.org/10.1504/IJWGS.2018.095647

[40] Zheng, Z., Xie, S., Dai, H., Chen, X. and Wang, H. (2017) An Overview of Blockchain Technology: Architecture, Consensus, and Future Trends. IEEE International Congress on Big Data, Honolulu, 25-30 June 2017, 557-564. https://doi.org/10.1109/BigDataCongress.2017.85

[41] Pilkington, M. (2016) Research Handbook on Digital Transformations. Edward Elgar.

[42] Yaga, D., Mell, P., Roby, N. and Scarfone, K. (2019) Blockchain Technology Overview, Cheltenham. https://doi.org/10.6028/NIST.IR.8202

[43] Gordon, W.J. and Catalini, C. (2018) Blockchain Technology for Healthcare: Facilitating the Transition to Patient-Driven Interoperability. Computational and Structural Biotechnology Journal, 16, 224-230. https://doi.org/10.1016/j.csbj.2018.06.003 